\title{
Laboratory-scale model of reinforced alkali-activated agro-waste for clayey soil stabilization
}

\begin{abstract}
This study assessed the laboratory model investigation to evaluate the feasibility of using reinforced alkali-activated binder for the purpose of soil stabilization. This paper consisted of two stages. The first stage investigated the efficacy of incorporating alkali-activated binder in conjunction with reinforcement inclusion, which improves satisfactory mechanical properties in treated soil. As such, in the first stage, palm oil fuel ash (POFA) and wollastonite microfibers were incorporated in the form of precursor and dispersed discrete reinforcement, respectively. In this stage, the unconfined compressive strength (UCS) was used as a practical indicator to investigate strength development. In the second stage of this study, a model procedure of interaction between a strip footing model and stabilized clayey soil by column technique was examined. From the perspective of strength development, alkali-activated agro-waste showed, undoubtedly, a very effective method to enhance the peak strength of investigated soil. Besides, the inclusion of the reinforcement material in alkali-activated samples increased the peak stress and improved the post-peak behavior, namely by modifying the original brittle response of the stabilized soil into a more ductile one. Depending on the replacement area ratio, results of the second phase showed that a considerable bearing capacity increase of up to $197 \%$ of treated columns could be achieved.
\end{abstract}

Keyword: Reinforcement materials; Wollastonite microfiber; Soil stabilization; Deep mixing method; Alkaline activation 